Irish Math. Soc. Bulletin

Number 72, Winter 2013, 71-74

ISSN 0791-5578

\title{
NESTING SYMMETRIC DESIGNS
}

\author{
PADRAIG Ó CATHÁIN
}

Arising from a problem concerning decomposition of graphs (see Section VI.24 of [3]), Darryn Bryant and Daniel Horsley posed the following problem:

Problem 1. Given a symmetric $(v, k, \lambda) \operatorname{design} \Delta$, when is it possible to add a point to each of the blocks of $\Delta$ to obtain a $\left(v, k+1, \lambda^{\prime}\right)$ design, $\Delta^{*}$ ?

We say that $\Delta$ can be nested if there exists a design $\Delta^{*}$ as in the problem. Nested triple systems have been considered in the literature: Stinson has shown that there exists a nested $(v, 3,1)$ if and only if $v \equiv 1 \bmod 6[6]$. We warn the reader that a related concept, also called nesting, involves the decomposition of a design with blocks of size $d k$ into $d$ designs with blocks of size $k$. Further details may be found in Section VI.36 of [3], but this problem will not be discussed here. In this note, we give a complete characterisation of nested symmetric designs. Our terminology for block designs is standard and follows, for example [1]. We remind the reader that a $(0,1)$ matrix $M$ is the incidence matrix of a symmetric $(v, k, \lambda)$ design if and only if $M M^{\top}=(k-\lambda) I+\lambda J$, where $I$ is the identity matrix, and $J$ is the all ones matrix (we omit subscripts for matrix orders, these can be determined from context). We refer the reader to [4] for an introduction to Hadamard matrices, and to [5] for a relatively recent survey of skew-Hadamard matrices.

Definition 2. A Hadamard matrix $H$ is skew-Hadamard (or skew) if

$$
H+H^{\top}=2 I
$$

Equivalently, $H-I$ is a skew-symmetric matrix.

2010 Mathematics Subject Classification. 05B05, 05B20.

Key words and phrases. Symmetric designs.

Received on 6-4-2013.

Support from the Australian Research Council through grant DP120103067 is gratefully acknowledged. 
We will require that a skew-Hadamard matrix has a skew-normal form. Denote by 1 a vector of 1 s of length $4 t-1$.

Lemma 3. Let $H$ be a skew-Hadamard matrix. Then $H-I$ is equivalent to a matrix of the form

$$
\left(\begin{array}{ll}
0 & 1 \\
-1^{\top} & M
\end{array}\right)
$$

where $M$ is skew. Furthermore, $M M^{\top}=(4 t-1) I-J$.

Proof. It suffices to observe that negating row $i$ and column $i$ of a skew matrix preserves the skew property.

To establish the claimed property of $M$, consider the matrix product

$$
(H-I)\left(H^{\top}-I\right)=\left(\begin{array}{ll}
0 & \mathbf{1} \\
-\mathbf{1}^{\top} & M
\end{array}\right)\left(\begin{array}{ll}
0 & -\mathbf{1} \\
\mathbf{1}^{\top} & M^{\top}
\end{array}\right)=(n-1) I .
$$

In particular, we see that $(-\mathbf{1})^{\top}(-\mathbf{1})+M M^{\top}=(n-1) I$. But $(-\mathbf{1})^{\top}(-\mathbf{1})=J$, and the result follows.

We recall that given a normalised Hadamard matrix, we obtain the incidence matrix of a $(4 t-1,2 t-1, t-1)$ design by deleting the first row and column of the Hadamard matrix and replacing -1 by 0 throughout. (See for example Lemma 7 of [2].) A similar operation can be applied to a Hadamard matrix where $H-I$ is in skew-normal form.

Lemma 4. Suppose that $H-I$ is in skew-normal form. Then $D=$ $\frac{1}{2}(M+J-I)$ is the incidence matrix of a $(4 t-1,2 t-1, t-1)$ design.

Proof. It suffices to show that $D D^{\top}=t I+(t-1) J$. We observe that the order of all matrices in the calculation below is $4 t-1$, that $M$ commutes with $J$, and that $M+M^{\top}=\mathbf{0}$. We calculate:

$$
\begin{aligned}
D D^{\top} & =\frac{1}{4}\left[M M^{\top}+\left(M+M^{\top}\right) J-\left(M+M^{\top}\right)+J^{2}-2 J+I\right] \\
& =\frac{1}{4}[(4 t-1) I-J+(4 t-1) J-2 J+I] \\
& =\frac{1}{4}[4 t I+(4 t-4) J]
\end{aligned}
$$

Hence $\frac{1}{2}(M+J-I)$ is the incidence matrix of a $(4 t-1,2 t-1, t-1)$ design as required. 
Definition 5. A design derived from a skew-Hadamard matrix as in Lemma 4 is a skew-design.

Lemma 6. Let $D$ be the incidence matrix of a skew-design with parameters $(4 t-1,2 t-1, t-1)$. Then $D+I$ is the incidence matrix of $a(4 t-1,2 t, t)$ design.

Proof. Observe first that $D+D^{\top}=\frac{1}{2}(M+J-I)+\frac{1}{2}\left(M^{\top}+J-I\right)=$ $J-I$. Then:

$$
(D+I)(D+I)^{\top}=D D^{\top}+(J-I)+I=t I+t J .
$$

Hence skew-designs are nested.

We conclude this note by showing that the nested property characterises skew-designs among all symmetric designs.

Theorem 7. A symmetric $(v, k, \lambda)$ design can be nested if and only if it is a skew-design.

Proof. (1) For any symmetric design we have that $\lambda=\frac{k(k-1)}{v-1}$. So for the statement of the theorem to hold, we require that $(v-1) \mid k(k-1)$ and $(v-1) \mid(k+1) k$. But then $v \mid$ $k(k+1)-k(k-1)$, or $v-1 \mid 2 k$. Since we can assume that $k \leq \frac{v}{2}$, we have that $v=2 k+1$, and $D$ has parameters $(4 t-1,2 t-1, t-1)$.

(2) Points added to distinct blocks must be distinct (because the replication number of a point is an invariant of a symmetric design).

(3) Skew-designs are nested by Lemma 6 .

(4) Let $M$ be the incidence matrix of $D$. Without loss of generality we order the blocks of the design (rows of the incidence matrix) so that the $i^{\text {th }}$ point is added to the $i^{\text {th }}$ block. So the incidence matrix of the new design is $M+I$. Now we require that

$$
(M+I)(M+I)^{\top}=t I+t J .
$$

But together with the requirement that $M M^{\top}=t I+(t-1) J$, this forces $M+M^{\top}=J-I$. So $2 M-J+I$ is a skew matrix, and $D$ is a skew-design. This completes the proof. 
P. Ó CATHÁIN

\section{REFERENCES}

[1] T. Beth, D. Jungnickel and H. Lenz: Design Theory. Vol I, Cambridge University Press, Cambridge, 1999.

[2] P. Ó Catháin: Difference sets and doubly transitive actions on Hadamard matrices, Journal of Combinatorial Theory, Series A, (6) 119, 2012, 12351249.

[3] C.J. Colbourn and J.H. Dinitz: Handbook of combinatorial designs, Chapman \& Hall/CRC, Boca Raton, FL, 2007.

[4] K.J. Horadam: Hadamard matrices and their applications, Princeton University Press, Princeton, NJ, 2007.

[5] C. Koukouvinos and S. Stylianou: On skew-Hadamard matrices, Discrete Mathematics, (13) 308 (2008), 2723-2731.

[6] D.R. Stinson: The spectrum of nested Steiner triple systems, Graphs and Combinatorics, (2) 1 (1985), 189-191.

Padraig Ó Catháin completed a PhD in algebraic design theory under the supervision of Dr Dane Flannery at the National University of Ireland, Galway in March 2012. Since September 2012 he has been employed as a postdoctoral researcher at the University of Queensland. His research interests are broadly in the interaction between algebra and combinatorics.

School of Mathematics and Physics, The University of QueEnsLAND, St LuCia, QLD 4072, Australia

E-mail address: p.ocathain@gmail.com 